\title{
El contexto PISA de los gráficos estadísticos en los libros de texto de matemáticas de Educación Básica en Costa Rica
}

\author{
| The PISA context of statistical graphics in basic education mathematics textbooks in Costa Rica |
}

\author{
Maynor Jiménez-Castro \\ mynor.jimenez@ucr.ac.cr \\ Universidad de Costa Rica \\ Guápiles, Costa Rica
}

\author{
José A. Garzón-Guerrero \\ jgarzon@ugr.es \\ Universidad de Granada \\ Granada, España
}

\author{
D Carmen Batanero \\ batanero@ugr.es \\ Universidad de Granada \\ Granada, España
}

\begin{abstract}
Resumen: El objetivo de este trabajo fue analizar los tipos de gráficos estadísticos y contextos descritos en las pruebas PISA asociados a los mismos en una muestra de libros de texto de matemáticas muy utilizados en la Educación General Básica en Costa Rica. El estudio contempla tres editoriales diferentes completas (un libro de texto para cada uno de los niveles de la educación primaria, niños de 7 a 11 años) y otras tres editoriales de educación secundaria (jóvenes de 13 a 15 años). En total, se analiza la distribución de 408 gráficos en los diferentes niveles de enseñanza y se comparan con resultados destacados en otras investigaciones. Igualmente se analizan los contextos en que se presentan los textos, utilizando la clasificación de los estudios PISA. Se constata un buen ajuste del tipo de gráfico respecto a las orientaciones curriculares y la presencia de los diferentes contextos, aumentando la presencia de las situaciones científicas en la educación secundaria.
\end{abstract}

Palabras Clave: educación básica, gráficos estadísticos, contexto, libros de texto

\begin{abstract}
This work aims to analyze the types of statistical graph and associated PISA contexts in mathematics textbooks directed to basic education in Costa Rica. The study includes three complete editorials with a book for each primary education level (7- 11 year old students) and another three editorials for secondary education levels (13-15 year-olds). In total, the distribution of 408 graphs at different levels of education is analyzed and compared with results of previous research. Likewise, the contexts in which the texts are presented are analyzed, using the classification of the PISA studies. There is a good fit of the type of graph, with respect to the curricular orientations and the different contexts are present, with an increasing presence of scientific contexts in secondary education.
\end{abstract}

Keywords: basic education, statistical graphs, context, textbooks

\section{Introducción}

La comprensión de gráficos estadísticos se hace cada vez más necesaria, debido a su frecuente presencia en los medios de comunicación y a la necesidad de interpretarlos para comprender las políticas

El contexto PISA de los gráficos estadísticos en los libros de texto de matemáticas de Educación Básica en Costa Rica. Jiménez, M., Garzón, J., Batanero. C.

Derechos Reservados @ 2022 Revista digital Matemática, Educación e Internet (https://revistas.tec.ac.cr/index.php/matematica) 
del estado y apoyar mejor la toma de decisiones. La importancia de su estudio ha llevado a las autoridades educativas en Costa Rica a introducirlos a lo largo de toda la educación básica (M.E.P., 2012), para dotar al futuro ciudadano del sentido estadístico que le permita una participación adecuada en la sociedad de la información (Batanero, 2019).

En las orientaciones curriculares actuales, los gráficos estadísticos se incluyen en el currículo costarricense de la educación básica, desde el $1^{\circ}$ al $9^{\circ}$ grado (estudiantes entre 6 y 15 años), de la siguiente forma (M.E.P., 2012):

- En primer grado, se destaca la importancia de la información cualitativa y cuantitativa y se promueve la recolección de datos como unidad básica para el trato de la información generada dentro de un contexto estudiantil. En este nivel los conceptos estadísticos se basan en el dato y recolección de información.

- En segundo grado, se introducen la lectura e interpretación de información resumida en dibujos, diagramas, cuadros y gráficos (se introduce el concepto de moda), con la finalidad de que se genere la capacidad de extraer información sencilla de los mismos;

- El trabajo con estos gráficos continúa en tercer grado con actividades de recolección, resumen y análisis de datos, así como también, la representación de resultados por medio de tablas y gráficos de barras y se introducen los conceptos de máximo y mínimo. Igualmente se plantea la habilidad para formular problemas simples que requieran la recolección de datos y su posterior análisis.

- En el cuarto año se inicia el II ciclo de la Educación General Básica (EGB) y con él se profundiza en la interpretación de información resumida en diferentes tipos de representación y generada en distintos contextos. Se introducen conceptos como los de media aritmética y recorrido y se promueven las gráficas de diagramas de puntos y lineales.

- En quinto grado, se enfatiza en las habilidades para reconocer la importancia del muestreo y la recolección de datos a través del cuestionario, así como también, en la elaboración gráficos de barras y circulares.

- En sexto año, se trabajan los diagramas lineales para visualizar las tendencias en series de tiempo. También se contempla la comparación gráfica de dos o más grupos de datos, trabajo que continúa en séptimo año.

- En séptimo año, que corresponde al inicio del tercer ciclo y la educación secundaria, se plantea la estadística como herramienta fundamental para la lectura y análisis de datos resumidos a través de diversas representaciones y aplicados en diferentes contextos. Se plantean actividades con gráficos, principalmente de barras.

- En octavo, se fomenta la recolección de datos y su representación en gráficos de barras, sectores, lineales y diagramas de puntos con frecuencias absolutas o relativas, con énfasis en el uso del software. Además, se utilizan los gráficos como apoyo en el estudio de la probabilidad.

- Finalmente, en noveno, se comienza la agrupación en intervalos y se representa la información utilizando histogramas o polígonos de frecuencia. Los gráficos también se utilizan en el estudio de la probabilidad frecuencial.

Estas orientaciones son similares a otras, como, por ejemplo, en Estados Unidos, Common Core State Standards for Mathematics (CCSSI, 2010) o España, Ministerio de Educación, Cultura y Deportes (MECD, 2014). Un requisito para cumplir los objetivos curriculares es la adecuada presentación del tema en los libros de texto utilizados por los estudiantes.

El contexto PISA de los gráficos estadísticos en los libros de texto de matemáticas de Educación Básica en Costa Rica. Jiménez, M.,

Garzón, J., Batanero. C.

Derechos Reservados @ 2022 Revista digital Matemática, Educación e Internet (https://revistas.tec.ac.cr/index.php/matematica) 


\section{Planteamiento del problema}

El objetivo del trabajo es analizar los tipos de gráficos introducidos en los diferentes grados de la educación general básica en algunas editoriales de libros de texto de uso frecuente en Costa Rica. Nos centramos en el libro de texto por constituir un paso intermedio entre las directrices curriculares y el currículo implementado en el aula (Herbel, 2007) y por su influencia en el aprendizaje del estudiante (Reys et al., 2004; van den Ham y Heinze, 2018). Este importante papel se refleja en el incremento de la investigación sobre el libro de texto de matemáticas (Fan et al., 2013).

Aunque ya existen algunos análisis de la presentación de los gráficos estadísticos en los libros de texto de matemáticas, que se describen en los antecedentes, estos estudios generalmente se limitan a la educación primaria y, salvo nuestro propio trabajo previo, se han llevado a cabo en países diferentes a Costa Rica. El estudio presentado en este trabajo pretende contribuir a llenar este vacío, para orientar a los profesores encargados de la enseñanza del tema y lograr un mejor aprendizaje de los gráficos estadísticos por parte de los estudiantes a lo largo de la educación básica en Costa Rica.

\section{Marco teórico}

\subsection{Antecedentes}

Son varios los autores que han estudiado los tipos de gráficos estadísticos que se presentan en los libros de texto, sobre todo desde que esta temática fue incluida en los currículos matemáticos en la educación primaria de sus respectivos países. Una de las investigaciones pioneras es la de Lemos (2006), quien analiza tres series de libros de texto de los cursos $1^{\circ}$ a $4^{\circ}$ de educación primaria en Brasil, mostrando que los gráficos en estos niveles y los libros analizados se reducen casi por completo al diagrama de barras.

Otro autor que ha realizado una investigación extensa es Díaz-Levicoy, casi siempre centrándose en la educación primaria. Uno sus estudios iniciales fueron llevados a cabo en tres series de libros de texto españoles y otras tres chilenas a lo largo de los seis cursos de educación primaria (Díaz-Levicoy et al., 2016). Al analizar los tipos de gráficos propuestos, los autores concluyen la alta frecuencia del gráfico de barras en todos los niveles curriculares analizados, con un porcentaje alrededor del $50 \%$ de todos los gráficos incluidos en ambos países en las series estudiadas. En los textos españoles, los siguientes más frecuentes son los gráficos de líneas $(21 \%)$ y sectores $(12 \%)$, mientras que en los chilenos serían los pictogramas $(22 \%)$ y de líneas y puntos ( $8 \%$ en cada caso).

Resultados similares se encontraron en el estudio de los gráficos estadísticos en libros de matemática de Educación Primaria de $4^{\circ}$ a $6^{\circ}$ curso en Argentina (Díaz-Levicoy, et al., 2017). En este caso, los gráficos de barras aparecen en un porcentaje del 29,5\% y con frecuencia muy similar los de sectores $(27 \%)$ y líneas $(25 \%)$. En otro trabajo con libros de texto peruanos (Díaz-Levicoy et al., 2018), se observa que prácticamente la mitad de los gráficos incluidos son diagramas de barras, seguidos por un $23 \%$ de gráficos de líneas y un $14 \%$ de pictogramas y gráficos de sectores (13\%).

Un precursor de este trabajo es el de Jiménez-Castro et al. (2020), donde se analizan los gráficos estadísticos en dos series de libros de texto de educación primaria ( $1^{\circ}$ a $6^{\circ}$ grado) en Costa Rica, En este trabajo se observaron diagramas de barras y lineales (simples y múltiples), diagrama de sectores, puntos y pictogramas, con gran predominio del diagrama de barras (57\%), seguido por los pictogramas $(15 \%)$.

El único antecedente encontrado con textos de secundaria es una investigación con dos libros chilenos de $7^{\circ}$ grado realizada por Díaz-Levicoy y Arteaga (2014), quienes indican que en dicho curso hay un predominio de los gráficos de barras y circulares y no se da atención a los gráficos de barras y líneas

El contexto PISA de los gráficos estadísticos en los libros de texto de matemáticas de Educación Básica en Costa Rica. Jiménez, M.,

Garzón, J., Batanero. C.

Derechos Reservados @ 2022 Revista digital Matemática, Educación e Internet (https://revistas.tec.ac.cr/index.php/matematica) 
múltiples, que apenas alcanzan el $10 \%$ de todos los gráficos.

Respecto al análisis del contexto de los gráficos en los libros de texto, encontramos el trabajo de Mateus (2014), quien estudia los gráficos en cinco libros de texto usados en educación básica en Colombia. Sus resultados muestran el predominio del contexto escolar, centrado en las vivencias en el aula, dejando de lado otras aplicaciones a disciplinas científicas. También se analizó esta variable en nuestro trabajo previo (Jiménez-Castro et al., 2020), en que se encontraron los cuatro contextos utilizados en el informe PISA con un peso aproximadamente igual.

El propósito de este trabajo es completar los anteriores, estudiando la distribución de los gráficos estadísticos y contextos utilizados en los niveles $1^{\circ}$ a $9^{\circ}$, es decir, estudiando a la vez la educación primaria y secundaria en Costa Rica y añadiendo una nueva serie de libros de textos a la ya analizada para la educación primaria.

\subsection{Bases teóricas}

\subsubsection{Comprensión gráfica}

La importancia de los gráficos se debe a su papel esencial en la organización y análisis de datos, pues al cambiar de un conjunto de datos brutos a un gráfico se crea nuevo conocimiento; por ejemplo, se visualiza la moda o las tendencias de los datos. Batanero et al. (2010), indican que incluso un gráfico elemental es un objeto semiótico complejo, cuya lectura o construcción requieren una serie de procesos interpretativos, de cada uno de sus elementos y del gráfico en conjunto. La comprensión gráfica implica capacidad para interpretar (en caso de lectura) o utilizar (si se trata de la construcción) los siguientes elementos de los gráficos (Curcio, 1987):

- El título, etiquetas de los ejes y de las escalas, que proporcionan las claves necesarias para comprender las variables representadas y sus relaciones.

- El contenido matemático subyacente; por ejemplo, los conjuntos numéricos empleados, los elementos geométricos, como longitud, proporcionalidad o área, que varían de un gráfico a otro.

- Los convenios utilizados para representar la información en el gráfico; por ejemplo, área en un histograma o altura en un diagrama de barras.

Friel et al. (2001), por su parte, describen las siguientes competencias relacionadas con la comprensión gráfica:

- Competencia para interpretar los diferentes componentes del gráfico (ejes, escalas, etiquetas, elementos específicos) y sus relaciones y para juzgar si dichos elementos se utilizan de forma correcta en un gráfico.

- Capacidad para predecir cómo influye en el gráfico cada una de estas componentes; por ejemplo, cómo cambiaría el gráfico al truncar la escala vertical.

- Contextualizar lo observado en el gráfico para poder hacer afirmaciones sobre los datos o variables representadas.

- Ser capaz de elegir un gráfico adecuado para un conjunto de datos dados, teniendo en cuenta la naturaleza de la variable y tamaño del conjunto de datos.

La inclusión de los gráficos en el currículo tiene como objetivo que los estudiantes adquieran estas competencias; un orden adecuado de introducción, desde los más simples a los más complejos será una condición indispensable para alcanzar este objetivo.

El contexto PISA de los gráficos estadísticos en los libros de texto de matemáticas de Educación Básica en Costa Rica. Jiménez, M.,

Garzón, J., Batanero. C.

Derechos Reservados @ 2022 Revista digital Matemática, Educación e Internet (https://revistas.tec.ac.cr/index.php/matematica) 


\subsubsection{El contexto en matemáticas y estadística}

Asistimos a un interés creciente por la importancia que tiene el contexto en educación matemática, puesto que la comprensión y resolución de problemas contextualizados forma parte de la cultura científica, es decir, del conjunto de conocimientos y competencias que necesita el ciudadano para desenvolverse en situaciones en que se requiere de conocimiento científico (Rosales et al., 2020). Los contextos variados atraen la atención de los estudiantes y conectan lo que aprende en la escuela con la sociedad externa a la misma, motivándole para seguir aprendiendo (Sanmartí y Márquez, 2017).

Dentro de la enseñanza de la matemática, la importancia del contexto ha crecido porque la sociedad exige vincular la acción del aula con la sociedad y debido a las pruebas PISA, que están teniendo una notable influencia en la preparación de los estudiantes en matemática en la mayoría de países (She et al., 2018). En dichas pruebas, más que evaluar los conocimientos de los estudiantes, se analiza su competencia para resolver problemas contextualizados en diversos tipos de situaciones cotidianas (Caraballo et al., 2013). Entre otras variables de las tareas propuestas en la evaluación, este programa tiene en cuenta el contexto de las tareas presentadas y diferencia varios tipos de contexto, que tendremos en cuenta en nuestro estudio.

\section{Marco metodológico}

\subsection{Concepción metodológica y tipo de investigación}

Se trata de una investigación cualitativa, donde se ha preferido estudiar con más detalle una muestra limitada de libros de texto y centrarnos únicamente en dos variables, para asegurar un proceso más sistemático y riguroso de investigación. Además, es exploratoria y descriptiva, puesto que el estudio de los gráficos en los libros de texto de Costa Rica o en los niveles de educación secundaria apenas ha sido abordado (Hernández et al., 2010).

Los datos que se analizan son documentos escritos, en concreto libros de texto, utilizando el análisis de contenido que permite identificar de forma sistemática y objetiva las características analizadas en un texto (Hernández-Sampieri y Torres, 2018). Para ello se completaron varias etapas:

- Determinar el objeto o tema de análisis, que fueron los gráficos estadísticos incluidos el texto.

- Determinar las reglas de codificación y categorías: se procede a identificar a priori a partir de investigaciones previas las categorías sobre tipo de gráfico, y contexto.

- Comprobar la fiabilidad del sistema de codificación-categorización: la codificación fue revisada independientemente por los autores, discutiendo los casos discordantes hasta llegar a un acuerdo.

\subsection{Muestra utilizada}

Para este estudio se han seleccionado tres series de libros de texto frecuentemente utilizadas en cada una de las etapas de educación primaria y secundaria de Costa Rica. En educación primaria se analizaron los correspondientes a la Asociación Libros para Todos del Grupo Nación (AL), Eduvisión (EV) y Editorial Santillana (SA). En educación secundaria, se revisaron los textos de las editoriales Publicaciones Innovadoras en Matemáticas (PI), Porras y Gamboa (PO) y Santillana (SA). En el anexo se presenta la lista completa de textos analizados.

Esta selección es intencional, más por el interés por profundizar en algunas características del uso de los gráficos estadísticos en los libros de texto dentro de la educación primaria y secundaria en

El contexto PISA de los gráficos estadísticos en los libros de texto de matemáticas de Educación Básica en Costa Rica. Jiménez, M.,

Garzón, J., Batanero. C.

Derechos Reservados @ 2022 Revista digital Matemática, Educación e Internet (https://revistas.tec.ac.cr/index.php/matematica) 
Costa Rica, que a establecer generalizaciones. La selección de la muestra es considerada dirigida o no probabilística (Hernández et al., 2010) del tipo opinático, referido al conocimiento que tiene el investigador de la situación o contexto en el que se aplican los libros de texto.

En cada uno de los libros de estas colecciones se examinaron todos los gráficos estadísticos incluidos, en total 408 gráficos cuya distribución se presenta en la Tabla 1.

Tabla 1: Distribución de gráficos analizados por editoriales en las dos etapas educativas

\begin{tabular}{ccccccccc}
\hline \multicolumn{3}{c}{ Editoriales Educación Primaria } & \multicolumn{4}{c}{ Editoriales Educación Secundaria } & $\begin{array}{c}\text { Total gráficos } \\
\text { analizados }\end{array}$ \\
\hline AL & SA & EV & Total Primaria & PI & PO & SA & Total Secundaria & \\
56 & 111 & 84 & 251 & 44 & 45 & 68 & 157 & 408 \\
\hline
\end{tabular}

\subsection{Variables analizadas}

Se consideraron dos variables independientes y dos variables dependientes. Como variables independientes se analizaron el grado escolar y la editorial, esta segunda separadamente para cada etapa de educación primaria y secundaria, puesto que se analizan editoriales diferentes en cada etapa, salvo una de ellas. También se tuvieron en cuenta dos variables dependientes:

- Tipo de gráfico: Se consideran todos los citados en las directrices curriculares y cualquier otro que aparezca en los libros de textos analizados.

- Contexto del gráfico. Para esta variable las categorías son las consideradas en los estudios PISA (OECD, 2016).

A continuación, se describen las dos variables dependientes, analizando un ejemplo en cada categoría considerada.

\subsubsection{Tipo de gráfico}

En los libros de textos analizados se encontraron los siguientes tipos de gráficos:

Gráficos de barras: utilizan barras, verticales u horizontales y de un mismo ancho para representar datos cualitativos o cuantitativos discretos. Están dispuestos en el primer cuadrante de un sistema cartesiano, en uno de los ejes se registra el valor numérico o modalidad de la variable y en el otro, las frecuencias, con una longitud proporcional de las barras. Se encuentran también variantes, como los gráficos de barras múltiples (ver Figura 1).
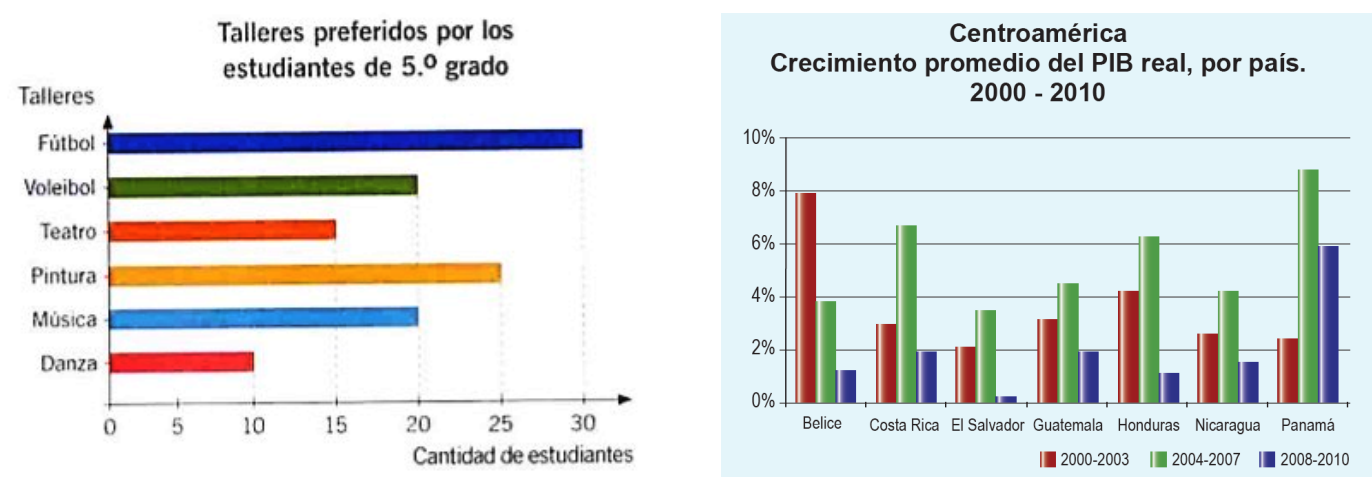

Figura 1: Gráficos de barras. Fuente: SA2, p.231 y PO7, p.103

El contexto PISA de los gráficos estadísticos en los libros de texto de matemáticas de Educación Básica en Costa Rica. Jiménez, M., Garzón, J., Batanero. C.

Derechos Reservados @ 2022 Revista digital Matemática, Educación e Internet (https://revistas.tec.ac.cr/index.php/matematica) 
Gráficos de líneas: es el gráfico en el que por lo general se utilizan puntos para representar la correspondencia entre el valor de la variable y su frecuencia de una variable en una serie de datos aislados y que se unen por medio de un segmento. Igual que en el caso anterior, este tipo de gráfico incluye gráficos de líneas múltiples que representan el comportamiento de dos o más variables a la vez. Ejemplos de estos gráficos en los cursos $4^{\circ}$ y $6^{\circ}$ se presentan en la Figura 2.
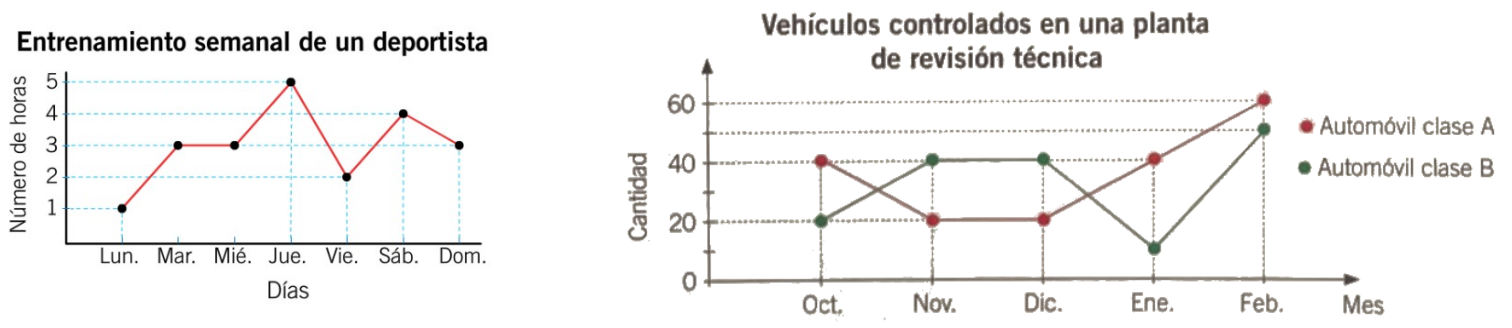

Figura 2: Ejemplo de gráficos de líneas. Fuente: SA4, p. 278 y SA6, p. 265

Pictograma: es un gráfico que utiliza íconos o imágenes del mismo tamaño, ordenados (horizontal o verticalmente) formando una especie de gráfico de barras, en las que las figuras alusivas al dato, representan una cantidad determinada de unidades. Un ejemplo del uso de pictogramas, lo encontramos en la Figura 3, donde cada imagen representada por una caja equivale a 3 kilogramos de cartón recolectados por la sección (grupo) de segundo grado respectivo.



Figura 3: Ejemplo de pictograma en $2^{\circ}$ grado. Fuente: SA2, p. 182

Diagramas de puntos: estos se pueden considerar un tipo particular de pictograma en los que los íconos o imágenes utilizadas para las frecuencias absolutas, corresponden a un punto, el cual se ubica sobre la columna (vertical $\mathrm{u}$ horizontal) tantas veces como lo indique la frecuencia de dicha variable. Un ejemplo se presenta en la Figura 4.

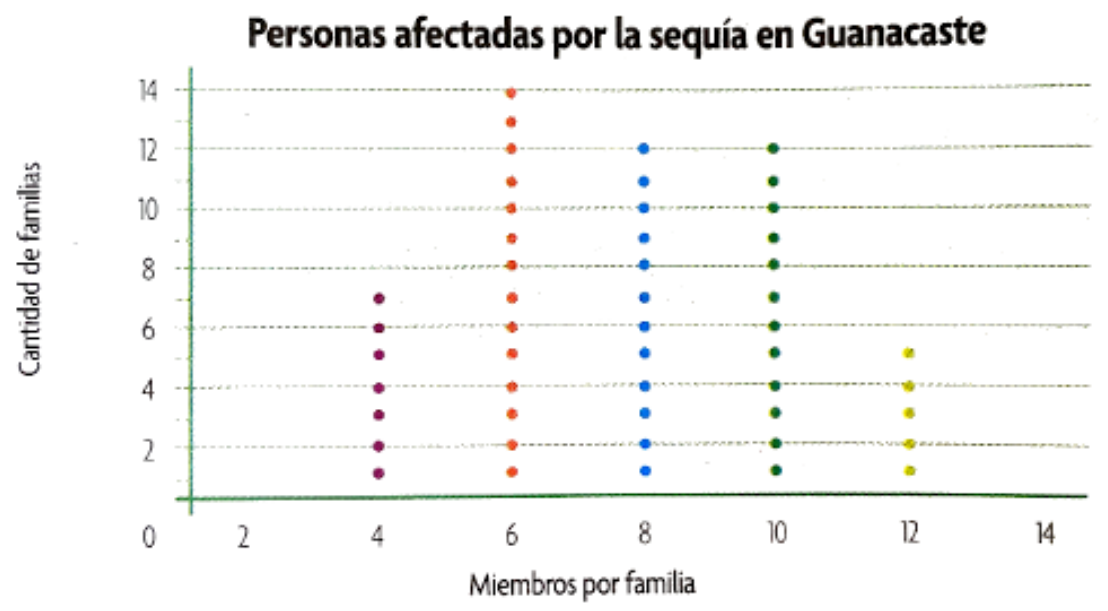

Figura 4: Ejemplo de diagramas de puntos utilizado en $4^{\circ}$ grado. Fuente: AL4, p. 68

El contexto PISA de los gráficos estadísticos en los libros de texto de matemáticas de Educación Básica en Costa Rica. Jiménez, M. Garzón, J., Batanero. C.

Derechos Reservados @ 2022 Revista digital Matemática, Educación e Internet (https://revistas.tec.ac.cr/index.php/matematica) 
Gráficos de sectores o circulares: es una representación utilizada comúnmente para destacar la parte de un conjunto de datos que representa cada categoría o valor de una variable. Cada una de ellas se representa mediante un sector circular de área, proporcional a la frecuencia relativa de la categoría en el conjunto de datos (Hernández, 2009). En el currículo costarricense, este tipo de gráficos se recomienda en II Ciclo a partir del $5^{\circ}$ y $6^{\circ}$ grado de la educación primaria. En la Figura 5 se muestra un ejemplo.



Figura 5: Ejemplo de gráfico de sectores para $5^{\circ}$ grado. Fuente: AL5, p. 152

Histogramas: cuando la variable cuantitativa es continua o con un número grande de valores, se agrupan sus valores para representar su distribución, tanto en una tabla como en un gráfico. El histograma es una representación gráfica de datos agrupados en intervalos, utilizando rectángulos, donde el área de cada intervalo es proporcional a la frecuencia de valores dentro del mismo (ver ejemplo, en la Figura 6).

La gráfica muestra la cantidad de habitantes de un país, agrupados por edad.

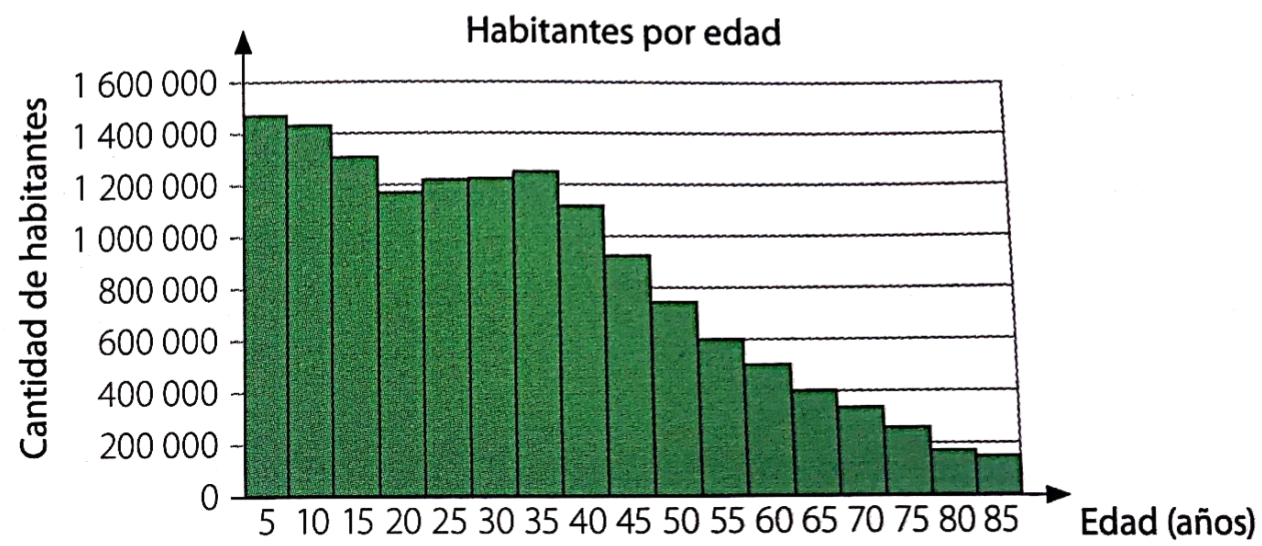

Figura 6: Ejemplo de histograma en $9^{\circ}$ año. Fuente: SA9, p. 136

Polígonos de frecuencias: es otra representación de datos agrupados en un intervalo. En este caso, se marca mediante un punto con coordenada x el punto medio del intervalo y ordenada y la frecuencia del intervalo a través de segmentos. Luego se unen todos los puntos. Aunque su aspecto es similar a un diagrama lineal, la principal diferencia en este caso es que la frecuencia representada se refiere a los datos de todo el intervalo y no a un valor aislado. Un ejemplo en $9^{\circ}$ año se presenta en la Figura 7. 




Figura 7: Ejemplo de polígono de frecuencias en 9o año. Fuente: SA9, p. 142

\subsubsection{Contexto del gráfico}

La segunda variable considerada ha sido el contexto de los datos presentados en el gráfico, que se han clasificado de acuerdo con los definidos en PISA (OECD, 2016), que se describen con detalle a continuación:

C1. Contexto personal: este contexto es común verlo ya que es muy cercano al estudiante y tomado de su vida diaria y por tanto es de relevancia directa e inmediata para la mayor parte de los estudiantes. Corresponde a situaciones en el ámbito de actividades personales del niño, de su familia o grupo de iguales. Un ejemplo de este contexto se presenta en la Figura 8, que muestra la puntuación dada por niños a diferentes tipos de actividades, según su preferencia.

- Observe y analice el siguiente diagrama.

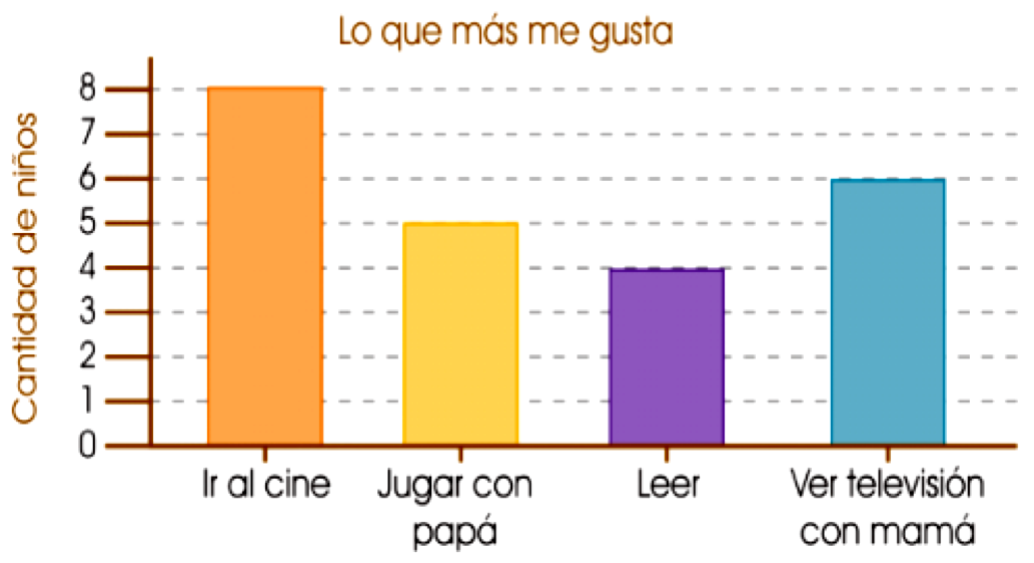

Figura 8: Ejemplo de contexto personal. Fuente: EV1, p. 272

C2. Contexto laboral o escolar: consiste en aquellas situaciones donde el problema aborda aspectos de la escuela o bien del mundo laboral de sus padres o su mundo laboral futuro. Por ejemplo, se representan variables relacionadas con la escuela, trabajo, empleo, producción o ventas. Un ejemplo se plantea en la Figura 1, donde el diagrama de barras izquierdo muestra los datos sobre los talleres opcionales que prefieren los estudiantes de un grupo del $5^{\circ}$ grado. La variable afecta no sólo al niño personalmente sino a un grupo mayor y se relaciona con el trabajo desarrollado en la escuela.

C3. Contexto social: son aquellas situaciones que se experimentan en las interacciones diarias con el mundo exterior y, por tanto, el foco de atención de la información representada es la comunidad (OECD , 2016). Corresponde a situaciones donde se aborda aspectos del ámbito social, más allá de la

El contexto PISA de los gráficos estadísticos en los libros de texto de matemáticas de Educación Básica en Costa Rica. Jiménez, M., Garzón, J., Batanero. C.

Derechos Reservados @ 2022 Revista digital Matemática, Educación e Internet (https://revistas.tec.ac.cr/index.php/matematica) 
vida personal del niño; por ejemplo, que pueden tener lugar en su barrio, su ciudad o país. Pueden presentar información sobre elecciones, transporte público, gobierno, demografía, publicidad, estadísticas nacionales o economía. También hemos incluido en este contexto los juegos de azar por ser ampliamente utilizados en la sociedad. Un ejemplo se muestra en la Figura 4, donde se representa el número de personas que componen las familias afectadas por una temporada de sequía en Guanacaste. El contexto excede la vida personal del estudiante y aunque está situado en una provincia particular de Costa Rica, el estudiante puede comprender que la sequía no es un problema que le afecta sólo como individuo, sino que perjudica a una comunidad más amplia.

C4. Contexto científico: este contexto se relaciona con problemáticas del ámbito de la ciencia y la tecnología e incluye aspectos como: el clima, ecología, medicina, ciencia espacial, genética, medición y el mundo de las matemáticas en sí. Reproducimos un ejemplo en la Figura 9, en que, mediante un diagrama de barras se presentan los porcentajes en que diversas fuentes contribuyeron a las emisiones de CO2 en Costa Rica en el año 2010. Este contexto es el más complejo, pues los estudiantes deben tener conocimientos sobre el $\mathrm{CO} 2$ y sus emisiones y efectos. Así se plantea en la actividad una serie de preguntas, de las cuáles las dos últimas requieren una capacidad de lectura crítica y la búsqueda de información no contenida en el gráfico por parte del estudiante.
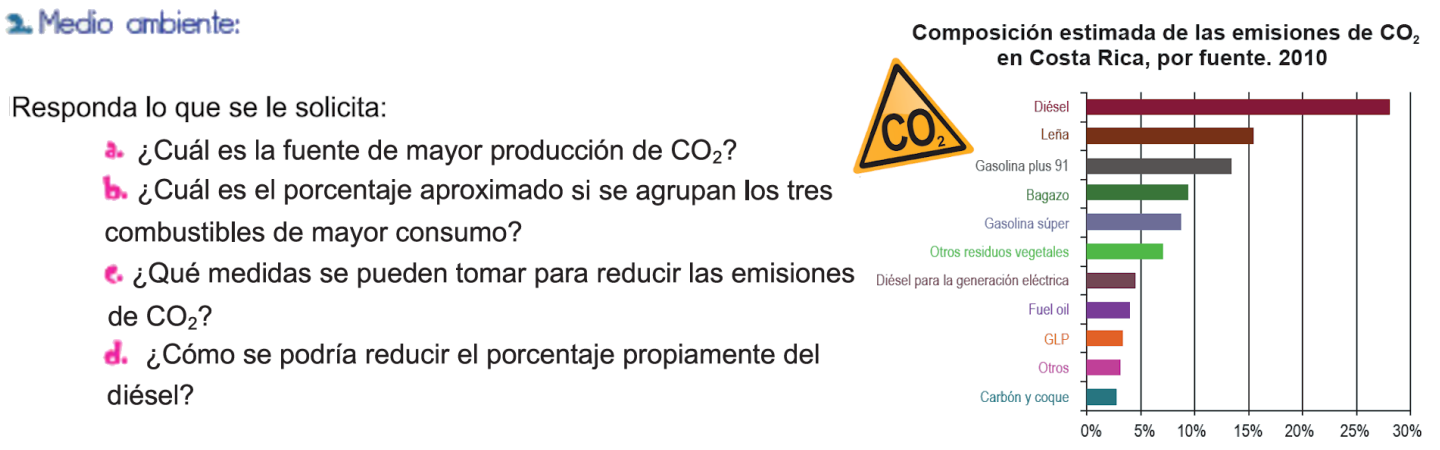

Fuente: Informe del estado de la nación XVIL Tomado de http:lummestaconacion. or

Figura 9: Ejemplo de contexto científico. Fuente: PO7, p.103

\section{Técnicas de procesamiento y análisis de datos}

El procesamiento de datos de esta investigación se realizó mediante el software SPSS y el análisis estadístico descriptivo. Luego de que los datos fueron codificados y registrados se desarrollaron las tablas de frecuencia reproducidas en el trabajo.

\section{Resultado del análisis de los gráficos}

\subsection{Gráficos presentados}

\subsubsection{Tipos de gráficos en los diferentes años escolares}

Al clasificar los gráficos propuestos en los libros de texto analizados, se han encontrado gráficos de barras, de líneas simples y múltiples, pictogramas, diagramas de puntos y gráficos de sectores (Tabla 2). Se observa el predominio del gráfico de barras, especialmente en los cursos de educación primaria ( $51 \%)$, lo que coincide con las investigaciones de Díaz-Levicoy et al. (2016), en que el gráfico de barras representa el $46 \%$ de las actividades en los textos españoles de educación primaria y el 42,5\% de los

El contexto PISA de los gráficos estadísticos en los libros de texto de matemáticas de Educación Básica en Costa Rica. Jiménez, M., Garzón, J., Batanero. C.

Derechos Reservados @ 2022 Revista digital Matemática, Educación e Internet (https://revistas.tec.ac.cr/index.php/matematica) 
chilenos e igualmente con Díaz-Levicoy et al. (2018) en el estudio realizado en el contexto peruano. En nuestro estudio, el único tipo de gráfico en el primer grado es el de barras y es el de mayor predominio hasta el tercer año.

Tabla 2: Distribución porcentual del tipo de gráfico por cada grado escolar en la Educación Básica. Nota: $n$ es el número de gráficos.

\begin{tabular}{|c|c|c|c|c|c|c|c|c|c|}
\hline \multirow[b]{2}{*}{$\begin{array}{l}\text { Tipo de } \\
\text { gráfico }\end{array}$} & \multicolumn{9}{|c|}{ Grado escolar } \\
\hline & $\begin{array}{c}1^{\circ} \\
(n=15)\end{array}$ & $\begin{array}{c}2^{\circ} \\
(\mathrm{n}=54)\end{array}$ & $\begin{array}{c}3^{\circ} \\
(n=46)\end{array}$ & $\begin{array}{c}4^{\circ} \\
(n=42)\end{array}$ & $\begin{array}{c}5^{\circ} \\
(\mathrm{n}=39)\end{array}$ & $\begin{array}{c}6^{\circ} \\
(\mathrm{n}=55)\end{array}$ & $\begin{array}{c}7^{\circ} \\
(\mathrm{n}=60)\end{array}$ & $\begin{array}{c}8^{\circ} \\
(n=50)\end{array}$ & $\begin{array}{c}9^{\circ} \\
(\mathrm{n}=47)\end{array}$ \\
\hline Barras & 100 & 68,5 & 73,9 & 33,3 & 59,0 & 9,1 & 43,3 & 32,0 & 10,6 \\
\hline $\begin{array}{l}\text { Barras múl- } \\
\text { tiples }\end{array}$ & & & & & & 14,5 & 11,7 & 6,0 & \\
\hline Líneas & & & 6,5 & 16,7 & & 38,2 & 8,3 & 16,0 & 8,5 \\
\hline $\begin{array}{l}\text { Líneas múl- } \\
\text { tiples }\end{array}$ & & & & & & 14,5 & 8,3 & 4,0 & 4,3 \\
\hline Pictogramas & & 31,5 & 6,5 & 7,1 & & & 3,3 & & \\
\hline Sectores & & & 13,0 & 4,8 & 28,2 & 14,5 & 25,0 & 24,0 & \\
\hline $\begin{array}{l}\text { Diagramas } \\
\text { de puntos }\end{array}$ & & & & 38,1 & 12,8 & 9,1 & & 18,0 & \\
\hline Histograma & & & & & & & & & 42,6 \\
\hline $\begin{array}{l}\text { Polígono de } \\
\text { frecuencias }\end{array}$ & & & & & & & & & 29,8 \\
\hline
\end{tabular}

El resto de gráficos se van utilizando según el grado escolar. Así, los pictogramas son ampliamente utilizados en segundo grado y luego prácticamente desaparecen. Por su lado, los diagramas de puntos son los más frecuentes en $4^{\circ}$ grado, pero disminuyen en $5^{\circ}$ y $6^{\circ}$ grados. En los textos argentinos (según la investigación de Díaz-Levicoy et al., 2016), se presenta una distribución más equitativa de los gráficos de barras, sectores y líneas en los cursos $4^{\circ}$ a $6^{\circ}$ de primaria, mientras en nuestro estudio se observa mayor variedad de gráficos en $6^{\circ}$ grado y todavía una fuerte presencia del gráfico de barras en $4^{\circ}$ y $5^{\circ}$ grados.

Las series analizadas incorporan mayor variedad de gráficos conforme se avanza el grado escolar. En primer año, sólo se presenta el gráfico de barras, en segundo se agregan los pictogramas y en tercero se suman gráficos de líneas y sectores circulares. En cuarto año, se agregan diagramas de puntos y en sexto grado se incorporan gráficos de barras y líneas múltiples. Estos distintos tipos de gráficos se mantienen en secundaria, pero en noveno año, su uso se concentra más en los histogramas y polígonos de frecuencia. No obstante, predomina el uso de gráficos de barras (y sus variantes) en los niveles de $7^{\circ}$ y $8^{\underline{o}}$ año, con una incorporación importante de los gráficos de sectores en estos dos niveles.

En el estudio de Díaz-Levicoy et al. (2016), centrado en la educación primaria, aparecen diagramas de barras y pictogramas en los dos primeros años en los textos chilenos, mientras los españoles se limitan a gráficos de barras. En los textos chilenos del tercer a quinto curso usados en este estudio, los gráficos de líneas son el segundo tipo más frecuente, mientras que en los libros costarricenses se utilizan muy poco en educación primaria y no tenemos datos de otros países para la educación secundaria. En los textos analizados de España y Chile se incorporan gráficos de líneas, pictogramas y sectores, a partir del cuarto año, mientras que en nuestro estudio estas representaciones gráficas se adelantan.

Por su lado, en el análisis de libros de secundaria, observamos una importante diferencia en relación con los estudios realizados en el tercer ciclo de la educación general básica de Portugal, por Jesus et al. (2013), donde la incidencia de gráficos circulares es mayor que en los libros costarricenses, donde el gráfico de barras (y sus variantes) acaparan la mayor frecuencia. En ambas investigaciones se da un trato especial a los gráficos de sectores en $7^{\circ}$ y $8^{\circ}$ año, dejando casi ausente su utilización en el $9^{\circ}$ año.

El contexto PISA de los gráficos estadísticos en los libros de texto de matemáticas de Educación Básica en Costa Rica. Jiménez, M., Garzón, J., Batanero. C. 
Tanto las orientaciones curriculares españolas (MECD, 2014), como las costarricenses (M.E.P, 2012), recomiendan los gráficos de barras, pictogramas y gráficos de sectores, como las principales gráficas en la educación primaria y para la educación secundaria, y además de estos, los histogramas y polígonos de frecuencia. Adicionalmente, el currículo básico español, incluye polígonos de frecuencias y la pirámide poblacional en educación primaria, que no son utilizados en el contexto costarricense, e histogramas en la educación secundaria. En el caso de Chile, el currículo escolar de primaria (MINEDUC, 2012), incluye diagramas de puntos, líneas y diagramas de tallo y hojas. Al comparar con lo propuesto en el currículo costarricense (M.E.P, 2012), se contemplan todos los gráficos indicados en los años correspondientes, excepto el gráfico de sectores, que la editorial Eduvisión lo adelanta al tercer año de primaria, cuando las orientaciones curriculares lo proponen para el quinto grado.

\subsubsection{Diferencias por editoriales}

En la Tabla 3 se presenta la distribución del tipo de gráfico en las diferentes editoriales analizadas en el estudio, separando las de educación primaria y secundaria.

Tabla 3: Distribución porcentual del tipo de gráfico en cada editorial. Nota: n es el número de gráficos.

\begin{tabular}{lcccccc}
\hline & \multicolumn{6}{c}{ Editoriales } \\
\cline { 2 - 7 } \multicolumn{1}{c}{ Tipo de gráfico } & EL & SA & EV & PI & PO & SA \\
& $(\mathrm{n}=56)$ & $(\mathrm{n}=111)$ & $(\mathrm{n}=84)$ & $(\mathrm{n}=44)$ & $(\mathrm{n}=45)$ & $(\mathrm{n}=68)$ \\
\hline Barras & 55,4 & 56,8 & 40,5 & 34,1 & 22,2 & 32,4 \\
Barras múltiples & 1,8 & 6,3 & & & 11,1 & 7,4 \\
Líneas & 10,7 & 5,4 & 22,6 & 15,9 & 11,1 & 7,4 \\
Líneas múltiples & 3,6 & 0,9 & 6,0 & 2,3 & 8,9 & 5,9 \\
Pictogramas & & 15,3 & 7,1 & 2,3 & & 1,5 \\
Sectores & 12,5 & 7,2 & 14,3 & 13,6 & 13,3 & 23,5 \\
Diagrama de puntos & 16,1 & 8,1 & 9,5 & 15,9 & 2,2 & 2,9 \\
Histograma & & & & 11,4 & 15,6 & 11,8 \\
Polígono de frecuencias & & & & 4,5 & 15,6 & 7,4 \\
\hline
\end{tabular}

En el caso de la educación primaria, los editoriales se enfocan en el uso primordialmente del gráfico de barras, sin embargo, EV (Eduvisón) también da énfasis a los gráficos de líneas y sectores, los cuales introduce desde el tercer año escolar, adelantándose a lo sugerido en las orientaciones curriculares.

De acuerdo con la etapa educativa, son las editoriales centradas en la educación secundaria las únicas que incluyen los histogramas y polígonos de frecuencia, ya que estos requieren del estudio de la agrupación de datos en intervalos. Estas editoriales continúan con una amplia presencia del gráfico de barras y también alguna presencia de casi todos los gráficos estudiados en la educación primaria. Hacemos notar también que los gráficos de barras y líneas múltiples quedan prácticamente restringidos a las editoriales dedicadas a la educación secundaria.

\subsection{Contextos presentados}

Se analizó el contexto en el cual se plantea un gráfico, utilizando la clasificación establecida en los estudios PISA (OECD, 2016), donde se plantea que el rendimiento matemático de un individuo puede ser influenciado por el contexto en el que desarrollen los problemas, que, por consiguiente, puede afectar la comprensión del gráfico estadístico.

El contexto PISA de los gráficos estadísticos en los libros de texto de matemáticas de Educación Básica en Costa Rica. Jiménez, M., Garzón, J., Batanero. C. 


\subsubsection{Tipos de contextos en los diferentes años escolares}

Los datos sobre el contexto se resumen en la Tabla 4 donde se muestra la relación del contexto con el nivel educativo. Todos los contextos citados en el informe PISA aparecen en todos los cursos escolares, excepto el concepto científico que no aparece en primer grado y tiene poca presencia hasta llegar al tercer año.

Tabla 4: Distribución porcentual de gráficos por tipo de contexto y año escolar.Nota: $n$ es el número de gráficos

\begin{tabular}{lccccccccc}
\hline \multirow{2}{*}{$\begin{array}{c}\text { Tipo de } \\
\text { Contexto }\end{array}$} & \begin{tabular}{c} 
G \\
\cline { 2 - 9 } \multicolumn{1}{c}{}
\end{tabular} & $2^{\circ}$ & $3^{\circ}$ & $4^{\circ}$ & $5^{\circ}$ & $6^{\circ}$ & $7^{\circ}$ & $8^{\circ}$ & $9^{\circ}$ \\
Personal & 60,0 & 37,0 & 39,1 & 21,4 & 7,7 & 16,4 & 13,3 & 30,0 & 23,4 \\
Escolar/laboral & 13,3 & 35,2 & 39,1 & 35,7 & 66,7 & 21,8 & 33,3 & 20,0 & 25,5 \\
Social & 26,7 & 18,5 & 19,6 & 21,4 & 10,3 & 36,4 & 35,0 & 38,0 & 34,0 \\
Científico & & 9,3 & 2,2 & 21,4 & 15,4 & 25,5 & 18,3 & 12,0 & 17,0 \\
\hline
\end{tabular}

En primer año el contexto preferente es el personal (60\%), lo que va de acorde a la experiencia del niño, incluyéndose también una proporción importante de contextos sociales. Dicho contexto personal va disminuyendo hasta el $5^{\circ}$ grado, aunque luego vuelve a subir, especialmente en $8^{\circ}$ y $9^{\circ}$ año. Ello es debido a la introducción de nuevos gráficos en estos cursos, donde entonces se ha preferido en el momento de la introducción utilizar contextos personales.

El contexto "laboral/escolar" aparece prácticamente en todos los niveles, sobre todo utilizando situaciones relacionadas con el trabajo en la escuela, pero con diferente intensidad. Es el contexto que predomina desde $3^{\circ}$ a $5^{\circ}$ grado, con un abordaje muy significativo en $2^{\circ}$ grado, mientras en Mateus (2014) predomina en todos los cursos.

El contexto social es el más representativo de los últimos niveles, es decir de $6^{\circ}$ a $9^{\circ}$ año, siguiendo un comportamiento similar al destacado en Mateus (2014), lo cual guarda una relación lógica por involucrar a los adolescentes en los problemas sociales y de vida en comunidad.

El contexto científico es escaso en los primeros años escolares, pero aumenta a partir del cuarto grado siguiendo las orientaciones curriculares planteadas desde el Ministerio de Educación Pública de Costa Rica (M.E.P, 2012).

\subsubsection{Diferencia por editoriales}

En la Tabla 5 se presenta la distribución del tipo de contexto que se utiliza en el gráfico en las diferentes editoriales analizadas en el estudio.

Tabla 5: Porcentaje de gráficos por tipo de contexto y editorial.Nota: n es el número de gráficos

\begin{tabular}{lcccccc}
\hline \multirow{2}{*}{$\begin{array}{c}\text { Tipo de } \\
\text { Contexto }\end{array}$} & \multicolumn{6}{c}{ Editoriales } \\
\cline { 2 - 7 } & AL & SA & EV & PI & PO & SA \\
& $(\mathrm{n}=56)$ & $(\mathrm{n}=111)$ & $(\mathrm{n}=84)$ & $(\mathrm{n}=44)$ & $(\mathrm{n}=45)$ & $(\mathrm{n}=68)$ \\
\hline Personal & 3,6 & 33,3 & 34,5 & 43,2 & 13,3 & 13,2 \\
Escolar /laboral & 48,2 & 33,3 & 33,3 & 22,7 & 28,9 & 27,9 \\
Social & 28,6 & 18,9 & 22,6 & 27,3 & 33,3 & 42,6 \\
Científico & 19,6 & 14,4 & 9,5 & 6,8 & 24,4 & 16,2 \\
\hline
\end{tabular}

El contexto PISA de los gráficos estadísticos en los libros de texto de matemáticas de Educación Básica en Costa Rica. Jiménez, M., Garzón, J., Batanero. C. 
En este caso se puede apreciar que todas las editoriales utilizan los diferentes contextos sugeridos en el estudio PISA, aunque hay diferencias entre ellas. Por un lado, la variación del porcentaje de gráficos con contexto personal es muy frecuente en las editoriales, hasta el punto de que algunas editoriales de educación secundaria (como PI) lo usan en mayor proporción que el resto de primaria. Ello se explica por qué dicha editorial apenas utiliza los contextos científicos, punto en que muestra una carencia, teniendo en cuenta que el libro va dirigido a estudiantes de primaria.

El contexto científico sigue siendo el menos frecuente, incluso en secundaria, en comparación con el resto de contextos, pero acá también observamos diferencias en porcentaje de gráficos desde el $6.8 \%$ al $24,4 \%$.

Globalmente, de acuerdo a la tabla, la editorial PO es la que presenta una distribución más uniforme y adecuada de contextos, al haber disminuido un poco el contexto personal (al tratarse de estudiantes de secundaria) y tener una proporción no muy diferente del resto de contextos.

\section{Conclusiones}

En este trabajo se han analizado diferentes gráficos estadísticos contenidos en libros de texto usados en Costa Rica, en las etapas de primaria y secundaria, así como los contextos asociados a los mismos. El estudio revela un buen ajuste entre los tipos de gráficos considerados por las editoriales analizadas en cada nivel educativo y lo sugerido en las directrices curriculares de Costa Rica (M.E.P., 2012). En concreto, el tipo de gráfico utilizado con mayor frecuencia en los libros de texto es el gráfico de barras, sin dejar de lado el uso de los otros gráficos indicados por los documentos curriculares en cada nivel escolar.

Los gráficos incrementan su dificultad al avanzar de curso, comenzando por la introducción del gráfico de barras en el primer año de primaria, añadiendo los pictogramas en segundo año, agregando los gráficos de sectores a partir del tercer grado y continuando con sectores y gráficos de puntos en cuarto e histogramas y polígonos de frecuencia en noveno. Esta propuesta metodológica responde fielmente a las orientaciones curriculares en cuanto a la incursión de los gráficos de barras y de los pictogramas, pero no así en cuanto al abordaje de otros tipos de gráficos. Así, el gráfico de sectores aparece en tercer grado, mientras que el currículum es propuesto para quinto curso y el histograma y los polígonos de frecuencias se adelantan también a octavo grado.

Los resultados obtenidos se asemejan a los obtenidos en otros estudios similares realizados en España, Chile, Argentina y Perú en cuanto al uso frecuente del diagrama de barras y presenta ligeras diferencias respecto a la presentación de otros gráficos en diferentes niveles educativos.

Respecto al estudio del contexto en el que se desarrollan los gráficos, los resultados muestran que varían conforme se avanza en los niveles educativos. En el primer año, los contextos "social" y "personal" son los más frecuentes. Posteriormente, las situaciones "laborales/escolares" se vuelven las más abundantes en las actividades de segundo a quinto año, notándose un incremento significativo del contexto "científico" desde cuarto a sexto año, convirtiéndose en este último curso en el tema más usual. Esta situación prácticamente coincide con un contexto "personal" en I ciclo, "escolar" en II ciclo $y$ "social" en III ciclo de la EGB en Costa Rica, reflejando coherencia con lo establecido en las recomendaciones curriculares dadas en (M.E.P, 2012), donde se indica la necesidad de incorporar aplicaciones matemáticas con temas cercanos al estudiante en primera instancia, pero introduciendo los contextos "escolar/laboral" y "social" posteriormente, sin dejar de lado las situaciones científicas en los niveles educativos superiores.

En resumen, las editoriales analizadas realizan un buen seguimiento de las recomendaciones y orientaciones curriculares emitidas por el Ministerio de Educación Pública, en cuanto a los tipos de gráfico y a los contextos en los que se utilizan, aunque en alguna de ellas se adelanta el uso de los gráficos de sectores al I ciclo de la EGB.

El contexto PISA de los gráficos estadísticos en los libros de texto de matemáticas de Educación Básica en Costa Rica. Jiménez, M.,

Garzón, J., Batanero. C.

Derechos Reservados @ 2022 Revista digital Matemática, Educación e Internet (https://revistas.tec.ac.cr/index.php/matematica) 
El carácter del estudio realizado es exploratorio, ya que no se han analizado libros de todas las editoriales costarricenses, pero contribuye a aportar información sobre el desarrollo curricular de los gráficos estadísticos a lo largo de toda la educación básica en Costa Rica, pues apenas se cuenta con investigaciones de este tipo para los cursos de la educación secundaria. En este sentido, el método y las categorías de análisis que han sido utilizadas en este trabajo pueden servir de base para el análisis de otros textos.

La información proporcionada es también valiosa para los profesores, pues se muestran dos variables de interés acerca de la enseñanza de los gráficos estadísticos, lo que puede permitir una mejor planificación del tema. El profesor puede apoyarse en el análisis para asegurar que todos los tipos de gráficos sugeridos en las directrices curriculares se incluyen en la enseñanza y asimismo tratar de utilizar contextos variados, incluyendo los propuestos en las pruebas PISA. Asimismo, al elegir un libro de texto sería recomendable analizar que estas dos variables tienen una adecuada distribución dentro del texto escogido.

Agradecimiento. Proyecto PID2019-105601GB-I00 (MICIN) y Grupo FQM-126 (Junta de Andalucía).

\section{Bibliografía}

[1] Batanero, C. (2019). Statistical sense in the information society. En: Villalba-Condori, K.O., Adúriz-Bravo, A., García-Peñalvo, F.J., Lavonen, J. (Eds.), Proceeding of the Congreso Internacional Sobre Educación y Tecnología en Ciencias - CISETC 2019 (pp. 28-38). Arequipa, Perú: CEUR-WS.

[2] Batanero, C., Arteaga, P. y Ruiz, B. (2010). Análisis de la complejidad semiótica de los gráficos producidos por futuros profesores de educación primaria en una tarea de comparación de dos variables estadísticas. Enseñanza de Las Ciencias, 28(1), 141-154.

[3] Caraballo, R. M., Rico, L. y Lupiáñez, J. L. (2013). Cambios conceptuales en el marco teórico competencial de PISA: el caso de las matemáticas. Profesorado, Revista de currículum y formación del profesorado, 17(2), 225-241.

[4] CCSSI (2010). Common Core State Standards for Mathematics. Washington: National Governors Association for Best Practices and the Council of Chief State School Officers.

[5] Curcio, F. R. (1987). Comprehension of mathematical relationships expressed in graphs. Journal for Research in Mathematics Education, 18(5), 382-393. https://doi.org/10.5951/ jresematheduc.18.5.0382.

[6] Díaz-Levicoy, D. y Arteaga, P. (2014). Análisis de gráficos estadísticos en textos escolares de séptimo básico en Chile. Diálogos Educativos, 14(28), 21-40.

[7] Díaz-Levicoy, D., Batanero, C., Arteaga, P. y Gea, M. M. (2016). Gráficos estadísticos en libros de texto de Educación Primaria: un estudio comparativo entre España y Chile. Bolema, 30(55), 713-737. https : //doi . org/10. 1590/1980-4415v30n55a20.

[8] Díaz-Levicoy, D., Giacomone, B. y Arteaga, P. (2017). Caracterización de los gráficos estadísticos en libros de texto Argentinos del segundo ciclo de educación primaria. Profesorado, 21(3), 299-326.

[9] Díaz-Levicoy, D., Osorio, M., Arteaga, P. y Rodríguez-Alveal, F. (2018). Gráficos Estadísticos en Libros de Texto de Matemática de Educación Primaria en Perú. Bolema, 32(61), 503-525. https://doi.org/10.1590/1980-4415v32n61a10.

El contexto PISA de los gráficos estadísticos en los libros de texto de matemáticas de Educación Básica en Costa Rica. Jiménez, M., Garzón, J., Batanero. C.

Derechos Reservados @ 2022 Revista digital Matemática, Educación e Internet (https://revistas.tec.ac.cr/index.php/matematica) 
[10] Fan, L., Zhu, Y. y Miao, Z. (2013). Textbook research in mathematics education: Development status and directions. Zentralblatt für Didaktik der Mathematik, 45(5), 633-646. https : //doi .org/10.1007/s11858-013-0539-x.

[11] Friel, S., Curcio, F. y Bright, G. (2001). Making sense of graphs: Critical factors influencing comprehension and instructional implications. Journal for Research in Mathematics Education, 32(2), 124-158. https://doi .org/10.2307/749671.

[12] Herbel, B. A. (2007). From intended curriculum to written curriculum: Examining the "voice" of a mathematics textbook. Journal for Research in Mathematics Education, 38(4), 344-369. https://www. jstor. org/stable/30034878.

[13] Hernández, O. (2009). Estadística elemental para ciencias sociales (3rd ed.). Universidad de Costa Rica.

[14] Hernández, R., Fernández, C. y Baptista, M. del P. (2010). Metodología de la Investigación. (5ta ed.). McGrawHill.

[15] Hernández-Sampieri, R. y Torres, C. P. M. (2018). Metodología de la investigación. McGrawHill

[16] Jiménez-Castro, M., Arteaga, P. y Batanero, C. (2020). Los gráficos estadísticos en los libros de texto de educación primaria en Costa Rica. Bolema, 34(66), 132-156. https : //doi . org/ $10.1590 / 1980-4415 v 34 n 66 a 07$.

[17] Jesus, D. S., Fernandes, J. A. y Leite, L. (2013). Relevância dos gráficos Eestatísticos nos manuais escolares da disciplina de ciências físico-químicas. In J. A. Fernandes, F. Viseu, M. H. Martinho y P. F. Correia (Eds.), Atas III Encontro de Probabilidades e Estatística na Escola (pp. 145-162). Centro de Investigação em Educação da Universidade do Minho.

[18] Lemos, M. P. F. D. (2006). O estudo do tratamento da informa ç ão nos livros didáticos das séries iniciais do Ensino Fundamental. Ciência \& Educação, 12(2), 171-184.

[19] Mateus, L. (2014). Estudio de gráficos estadísticos usados en una muestra de libros de matemáticas para la educación básica y media en Bogotá. En L. Andrade (Ed.), I Encuentro Colombiano de Educación Estocástica (pp. 274-280). Bogotá.

[20] MECD (2014). Real Decreto 126/2014, de 28 de febrero, por el que se establece el currículo básico de la educación primaria. Autor.

[21] M.E.P., Ministerio de Educación Pública (2012). Programa de Estudio Matemáticas, I, II y III Ciclos de la Educación General Básica y Ciclo Diversificado. Ministerio de Educación Pública.

[22] MINEDUC (2012). Matemática educación básica. Bases curriculares. Santiago: Autor.

[23] OECD (2016). PISA 2015 assessment and analytical framework: Science, reading, mathematic and financial literacy. OECD Publishing.

[24] Rosales, E. M., Rodríguez, P. y Romero, M. (2020). Conocimiento, demanda cognitiva y contextos en la evaluación de la alfabetización científica en PISA. Eureka 17(2), 2302. https://doi.org/10.25267/Rev_Eureka_ensen_divulg_cienc.2020.v17.i2.2302.

[25] Reys, B. J., Reys, R. E. y Chavez, O. (2004). Why mathematics textbooks matter. Educational Leadership, 61(5), 61-66.

[26] Sanmartí, N. y Márquez, C. (2017). Aprendizaje de las ciencias basado en proyectos: del contexto a la acción. Ápice, 1(1), 3-16.

El contexto PISA de los gráficos estadísticos en los libros de texto de matemáticas de Educación Básica en Costa Rica. Jiménez, M., 
[27] She, H. C., Stacey, K. y Schmidt, W. H. (2018). Science and mathematics literacy: PISA for better school education. International Journal of Science and Mathematics Education, 16(1), $1-5$.

[28] van den Ham, A. K. y Heinze, A. (2018). Does the textbook matter? Longitudinal effects of textbook choice on primary school students' achievement in mathematics. Studies in Educational Evaluation, 59, 133-140.

\section{A. Apéndice}

\section{A.1. Textos usados en el análisis y códigos utilizados en los ejemplos.}

\section{Textos de educación primaria}

AL1. Asociación Libros para Todos. (2017). Matemática 1. (Y. Calderón Quesada, Ed.) (5th ed.). GN Impresos.

AL2. Asociación Libros para Todos. (2017). Matemática 2. (Y. Calderón Quesada, Ed.) (5th ed.). GN Impresos.

AL3. Asociación Libros para Todos. (2017). Matemática 3. (Y. Calderón Quesada, Ed.) (5th ed.). GN Impresos.

AL4. Asociación Libros para Todos. (2017). Matemática 4. (Y. Calderón Quesada, Ed.) (5th ed.). GN Impresos.

AL5. Asociación Libros para Todos. (2017). Matemática 5. (Y. Calderón Quesada, Ed.) (5th ed.). GN Impresos.

AL6. Asociación Libros para Todos. (2017). Matemática 6. (Y. Calderón Quesada, Ed.) (5th ed.). GN Impresos.

SA1. Santillana. (2016). Matemática 1. (Editorial Santillana, Ed.) (1st ed.).

SA2. Santillana. (2016). Matemática 2. (Editorial Santillana, Ed.) (1st ed.).

SA3. Santillana. (2016). Matemática 3. (Editorial Santillana, Ed.) (1st ed.).

SA4. Santillana. (2016). Matemática 4. (Editorial Santillana, Ed.) (1st ed).

SA5. Santillana. (2016). Matemática 5. (Editorial Santillana, Ed.) (1st ed.).

SA6. Santillana. (2016). Matemática 6. (Editorial Santillana, Ed.) (1st ed

EV1. Eduvisión. (2014). Visión Matemática 1 (1st ed.). Eduvisión.

EV2. Eduvisión. (2014). Visión Matemática 2 (1st ed.). Eduvisión.

EV3. Eduvisión. (2014). Visión Matemática 3 (1st ed.). Eduvisión.

EV4. Eduvisión. (2014). Visión Matemática 4 (1nd ed.). Eduvisión.

EV5. Eduvisión. (2014). Visión Matemática 5 (1st ed.). Eduvisión.

EV6. Eduvisión. (2015). Visión Matemática 6 (2nd ed.). Eduvisión.

\section{Textos de educación secundaria}

PI7. Publicaciones Innovadoras en Matemáticas para Secundaria (2017). Matemática 7. Autor. PI8. Publicaciones Innovadoras en Matemáticas para Secundaria (2017). Matemática 8. Autor. PI9. Publicaciones Innovadoras en Matemáticas para Secundaria. (2017). Matemática 9. Autor. PO7. Publicaciones Porras y Gamboa (2017). Matemática 7. Autor. PO8. Publicaciones Porras y Gamboa (2017). Matemática 8. Autor. PO9.Publicaciones Porras y Gamboa (2017). Matemática 9. Autor.

SA7. Santillana (2017). Matemática 7. Autor.

SA8. Santillana (2017). Matemática 8. Autor.

SA9. Santillana (2017). Matemática 9. Autor.

El contexto PISA de los gráficos estadísticos en los libros de texto de matemáticas de Educación Básica en Costa Rica. Jiménez, M., Garzón, J., Batanero. C. 